\title{
Análise de Desempenho de um Roteador utilizando Diferentes Arquiteturas de Decremento em uma Rede-em-Chip
}

T. de OLIVEIRA ${ }^{1}$, Departamento de Engenharia Elétrica, FEIS, UNESP, 15385-000 Ilha Solteira, SP, Brasil.

N. MARRANGHELLO ${ }^{2}$, Departamento de Ciências de Computação e Estatística, IBILCE, UNESP, 15054-000 São José do Rio Preto, SP, Brasil.

\begin{abstract}
Resumo. Uma rede-em-chip está sendo desenvolvida para permitir a implementação de uma Rede de Petri em hardware. Para determinar a melhor arquitetura do decrementador a ser incorporada ao roteador dessa rede foi desenvolvida uma abordagem baseada em equações matemáticas que computam as quantidades de portas lógicas e de níveis de lógica dos decrementadores e do roteador. Uma fórmula de desempenho foi estabelecida para realizar uma análise comparativa da arquitetura do roteador com cada um dos decrementadores.
\end{abstract}

Palavras-chave. Roteador, decrementadores, desempenho.

\section{Introdução}

Devido a um mercado atual bastante competitivo, o projeto de sistemas digitais tem se tornado cada vez mais complexo e exigindo um tempo de confecção bastante curto. Diante deste panorama, vem surgindo uma necessidade crescente de ferramentas CAD que possam auxiliar os projetistas na tomada de decisão durante o desenvolvimento de um sistema. Análises realizadas antes da etapa de implementação física podem economizar custos e melhorar o desempenho do sistema em relação ao consumo de energia, tempo de resposta entre outros fatores. Com isso, algumas técnicas têm sido propostas para a estimativa da área, tempo de resposta e consumo de energia de um dado sistema digital. Ramachandran et al. [6] realizam uma estimativa da área e do tempo de resposta de um sistema baseando-se em modelos com arquiteturas de layouts pré-definidas. Srinivasan et al. [7] estimam a área e o tempo de resposta total do sistema utilizando informações provenientes da síntese lógica de apenas um subconjunto de circuitos do projeto. Nemani e Najm [5] realizam uma estimativa da área e do consumo de energia baseando-se na quantidade e no tamanho dos implicantes primos das funções booleanas que compõem

\footnotetext{
${ }^{1}$ tiagoooli@yahoo.com.br

${ }^{2}$ norian@ibilce.unesp.br
} 
o sistema. Büyüksahin e Najm [1] descrevem o sistema por meio de uma rede booleana e, a extração de determinadas características dessa rede, como a quantidade de nós, a quantidade de arcos e o grau dos nós permite uma estimativa da área do sistema. Gelosh e Steliff [3] utilizam técnicas de aprendizado de máquina para modelar a própria ferramenta de síntese e utilizar esse modelo para a obtenção da área e do tempo de resposta do sistema.

Imagine-se um sistema digital composto por vários blocos digitais, precisando executar uma determinada tarefa a qual pode ser implementada por meio de arquiteturas diferentes. Este artigo procura identificar, para o projeto de um roteador numa rede-em-chip, a melhor arquitetura que deve ser implementada em cada bloco funcional para que a rede-em-chip como um todo atinja o seu desempenho máximo. Além disso, numa possível expansão dos sinais de entrada/saída do roteador, essas equações matemáticas podem ser reutilizadas para determinar a configuração que deve ser adotada para se obter o desempenho estabelecido.

\section{Motivação deste Trabalho}

Baseando-se nos conceitos de rede-em-chip [4], nosso grupo de projeto desenvolveu em VHDL um roteador capaz de determinar o trajeto de um pacote de dados sobre uma rede-em-chip com uma topologia de malha bidimensional. Na arquitetura do roteador projetado foi necessário o uso de estruturas aritméticas (decrementadores) para computar o endereço de destino de um pacote. A rede-em-chip desenvolvida recebe pacotes contendo, em seu cabeçalho, alguns campos referentes ao endereço de destino (variações nos eixos x e y). Ao receber um pacote, o roteador verifica os valores armazenados nos campos de variações. Se forem zeros, o pacote chegou a seu destino. Caso contrário, um campo de variação é decrementado em uma unidade e o pacote é enviado a um roteador vizinho.

Arquiteturas diferentes podem ser utilizadas nesses decrementadores levando em consideração a forma de obtenção do bit de transporte. Dependendo da arquitetura utilizada para esses decrementadores o roteador pode alcançar uma velocidade maior de processamento ou/e uma área menor numa possível implementação física da arquitetura proposta num chip ou num dispositivo lógico programável. Uma maior velocidade de execução do sistema de comunicação, composto por um conjunto de roteadores, diminuirá o tempo de resposta da rede-em-chip proposta. Por sua vez, a redução da área de cada roteador pode reduzir o custo da fabricação em um circuito integrado da rede proposta.

A abordagem apresentada neste artigo tem como propósito comparar o desempenho do roteador com as principais e mais conceituadas técnicas de obtenção do sinal de transporte, quais sejam, decrementadores com transporte em cascata, decrementadores com transporte antecipado e decrementadores com transporte selecionado e identificar a melhor técnica que deve ser utilizada no projeto do roteador.

Na próxima seção, especificam-se os conceitos fundamentais e as equações matemáticas que possibilitam a comparação de desempenho da arquitetura do roteador utilizando, como exemplo, o decrementador com transporte antecipado. Posteriormente, apresentam-se os resultados obtidos sobre o desempenho do roteador para cada decrementador, finalizando, com alguns comentários e discussões sobre os re- 
sultados obtidos.

\subsection{Decrementador com transporte antecipado}

O decrementador com transporte antecipado [2] é capaz de determinar os bits de transporte de todos módulos de subtração utilizando apenas os sinais de entrada dos operandos (X e Y) e o vem-um inicial $\left(C_{0}\right)$. Com isso, o cálculo dos bits de transporte é realizado simultaneamente, não necessitando realizar a propagação do vai-um como acontece no decrementador com transporte em cascata. A figura 1 mostra a arquitetura e o caminho crítico do decrementador com transporte antecipado. O circuito gerador de transporte antecipado determina os valores de todos os bits de transporte intermediários antes que os bits correspondentes da subtração sejam computados. O circuito gerador pode ser equacionado da seguinte forma

$$
\begin{aligned}
C_{1}= & 0+P_{0} \\
C_{2}= & G_{1}+0+P_{1} \cdot P_{0} \\
\vdots & \vdots \\
C_{n}= & G_{n-1}+P_{n-1} \cdot G_{n-2}+P_{n-1} \cdot P_{n-2} \cdot G_{n-3}+P_{n-1} \cdot P_{n-2} \cdot P_{n-3} \cdot G_{n-4} \\
& +\ldots+P_{n-1} \cdot P_{n-2} \cdot P_{n-3} \ldots P_{2} \cdot G_{1}+0+P_{n-1} \cdot P_{n-2} \ldots P_{0} .
\end{aligned}
$$

Com relação à quantidade de níveis de lógica, pode ser observado que os sinais auxiliares $P$ e $G$ precisam de no máximo um nível de lógica, correspondente ao atraso de uma porta lógica not. Para a produção da subtração, de acordo com a fórmula $S_{i}=P_{i} \oplus C_{i}$, é necessário o atraso de mais uma porta lógica.

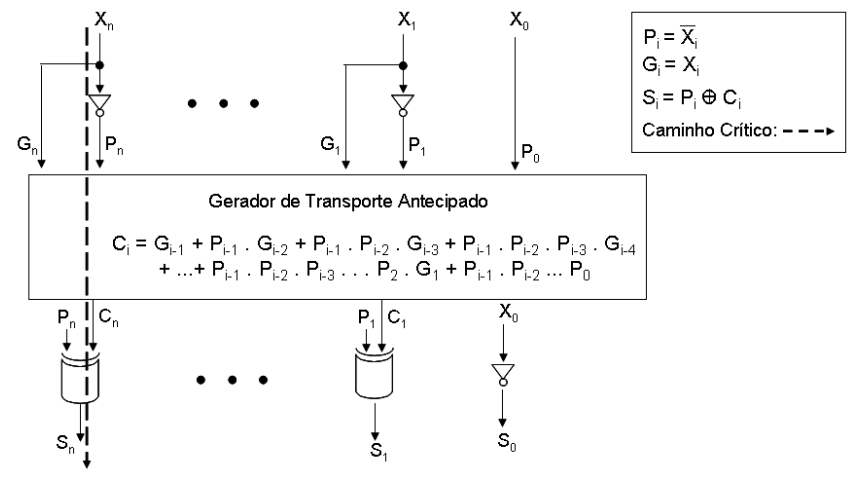

Figura 1: Caminho crítico para o decrementador com transporte antecipado

O circuito gerador de transporte antecipado realiza o cálculo dos sinais $C_{i}$ em paralelo e, portanto, apenas o atraso do bit de transporte do último nível deve ser levado em consideração, visto que este sinal de transporte possui a expressão booleana que gera o maior atraso. Tomando $n+1$ como o índice de maior nível, tem-se

$$
C_{n+1}=G_{n}+P_{n} \cdot G_{n-1}+P_{n} \cdot P_{n-1} \cdot G_{n-2}+P_{n} \cdot P_{n-1} \cdot P_{n-2} \cdot G_{n-3}+\ldots+
$$




$$
+P_{n} \cdot P_{n-1} \cdot P_{n-2} \ldots P_{2} \cdot G_{1}+0+\underbrace{P_{n} \cdot P_{n-1} \ldots P_{0}}_{\text {maior atraso }} .
$$

Note-se que para a geração do sinal $C_{n+1}$, os mintermos (termos de produtos, representado operações and) são realizados simultaneamente e, portanto, deve-se levar em consideração apenas o mintermo com maior atraso, ou seja, o mintermo que possuir um maior número de operandos. Na expressão anterior, o mintermo de maior atraso, possui $(n+1)$ entradas, que corresponde a um atraso de $\left\lceil\log _{2}(n+1)\right\rceil$ níveis de lógica. Após a execução da operação lógica and, deve-se computar o atraso máximo gerado pela realização das operações lógicas or, visto que os mintermos e as operações booleanas or são realizadas seqüencialmente. Na expressão anterior para o sinal $C_{n+1}$, as operações lógicas or terão $(n+1)$ operandos de entrada, visto que $C_{n+1}=G_{n}+\ldots+G_{n-1}+\ldots G_{n-2}+\ldots G_{1}+P_{n} \cdot P_{n-1} \ldots P_{0}$. Portanto, para a realização das operações or será necessário um atraso máximo de $\left\lceil\log _{2}(n+1)\right\rceil$ níveis de lógica.

Assim, a fórmula completa indicando a quantidade de níveis de lógica da arquitetura do decrementador com transporte antecipado será

$$
\begin{aligned}
N L & =\left\lceil\log _{2}(n+1)\right\rceil+\left\lceil\log _{2}(n+1)\right\rceil+2 \\
& =2 *\left\lceil\log _{2}(n+1)\right\rceil+2 .
\end{aligned}
$$

Com relação à quantidade de portas lógicas do decrementador com transporte antecipado, primeiro, será calculada a quantidade de portas do gerador de transporte antecipado e depois, serão computadas as lógicas de produção dos sinais auxiliares $P$ e $G$, e subtração. De acordo com a equação 2.1, a quantidade de portas lógicas $(Q P)$ necessárias para implementar a lógica do sinal $C_{1}$ é zero, para o sinal $C_{2}$ é 2 (uma operação lógica and entre $P_{1}$ e $P_{0}$ e uma operação lógica or entre os operandos $G_{1}$ e o resultado da operação and), assim $Q P\left(C_{1}\right)=0, Q P\left(C_{2}\right)=2$, $Q P\left(C_{3}\right)=5, Q P\left(C_{4}\right)=9$ e $Q P\left(C_{5}\right)=14$.

O sinal $C_{n}$ possui $n$ operandos para a realização da operação binária or, visto que:

$$
C_{n}=\underbrace{G_{n-1}+\ldots G_{n-2}+\ldots G_{n-3}+\ldots G_{n-4}+\ldots+\ldots G_{1}}_{n-1 \text { operandos }}+P_{n-1} \ldots P_{0} .
$$

Assim, para a realização da operação or são necessárias $(n-1)$ portas lógicas or de duas entradas. O sinal $C_{n}$ ainda possui $(n-1)$ termos contendo operações and, como mostrado na equação 2.1. A cada termo and, da esquerda para a direita da expressão de $C_{n}$, acrescenta-se um operando a mais, como mostra a tabela 1.

Assim, de acordo com a tabela 1, para a realização de todas as operações and em todos os termos do sinal $C_{n}$ são necessárias $\sum_{i=1}^{n-1} i$ portas lógicas and de duas entradas. Desta forma, tem-se

$$
Q P\left(C_{n}\right)=\underbrace{(n-1)}_{\text {portas or }}+\underbrace{\sum_{i=1}^{n-1} i}_{\text {portas and }} .
$$


Tabela 1: Acréscimo de portas lógicas de um termo para outro

\begin{tabular}{|c|c|c|}
\hline Termo & Quantidade de portas & Correspondência \\
\hline \hline 1 & 1 & $P_{n-1} \cdot G_{n-2}$ \\
\hline 2 & 2 & $P_{n-1} \cdot P_{n-2} \cdot G_{n-3}$ \\
\hline 3 & 3 & $P_{n-1} \cdot P_{n-2} \cdot P_{n-3} \cdot G_{n-4}$ \\
\hline 4 & 4 & $P_{n-1} \cdot P_{n-2} \cdot P_{n-3} \cdot P_{n-4} \cdot G_{n-5}$ \\
\hline$\vdots$ & $\vdots$ & $\vdots$ \\
\hline$(n-1)$ & $(n-1)$ & $P_{n-1} \cdot P_{n-2} \ldots P_{0}$ \\
\hline
\end{tabular}

A quantidade total de portas lógicas do gerador de transporte antecipado (GTA) pode então ser expressa da seguinte maneira:

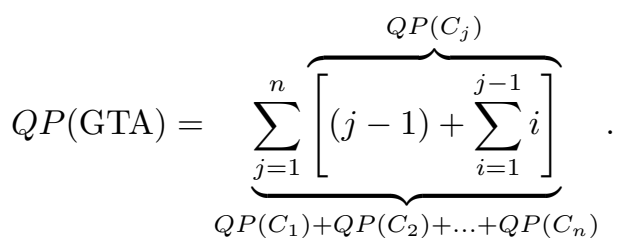

Expandindo, tem-se $Q P(\mathrm{GTA})=\sum_{j=1}^{n} \sum_{i=1}^{j-1} i+\sum_{j=1}^{n} j-n$.

Para finalizar o cálculo da quantidade de portas do decrementador com transporte antecipado, é necessário computar a quantidade de portas lógicas dos sinais auxiliares $P$ e $G$, e subtração.

Como mostrado na figura $1, P_{0}=X_{0}, G_{0}=0$ e $S_{0}=\overline{X_{0}}$ e assim, para a geração dos sinais auxiliares e subtração do primeiro bit, será necessário apenas uma porta lógica.

Para os demais $P_{i}, G_{i}$ e $S_{i}$, tem-se $P_{i}=\overline{X_{i}}, G_{i}=X_{i}$ e $S_{i}=P_{i} \oplus C_{i}$. Assim, para cada $\left(P_{i}, G_{i}\right.$ e $\left.S_{i}\right)$ precisa-se de duas portas lógicas.

Portanto, tomando $n$ como sendo o tamanho (quantidade de bits) do decrementador com transporte antecipado, a quantidade de portas lógicas para todos os sinais auxiliares e subtrações é dada por $Q P(\mathrm{P}, \mathrm{G}$ e $\mathrm{S})=1+2 * n$.

A quantidade de portas lógicas do decrementador com transporte antecipado é dada por: $Q P=Q P(\mathrm{GTA})+Q P(\mathrm{P}, \mathrm{G}$ e $\mathrm{S})$. Substituindo, tem-se

$$
Q P=\left(\sum_{j=1}^{n} \sum_{i=1}^{j-1} i+\sum_{j=1}^{n} j-n\right)+(1+2 * n) .
$$

\section{Fórmula de Desempenho}

A fórmula de desempenho envolvendo a quantidade de portas lógicas (QP) e a quantidade de níveis de lógica (NL) pode ser assim esquematizada

$$
D E=P_{q p} *\left(\frac{Q P_{\min }}{Q P}\right)+P_{n l} *\left(\frac{N L_{\min }}{N L}\right),
$$


onde $P_{q p}$ indica o peso dado ao desempenho em relação à quantidade de portas lógicas $(\mathrm{QP})$ e $P_{n l}$ indica o peso dado ao desempenho em relação à quantidade de níveis de lógica (NL). Os valores atribuídos aos pesos $P_{q p}$ e $P_{n l}$ devem satisfazer a seguinte relação: $P_{q p}+P_{n l}=1$.

Esses pesos permitem um ajuste fino na análise matemática do sistema, o que pode permitir, por exemplo, que durante o processo de análise haja uma maior preocupação com a quantidade de níveis de lógica do sistema correspondente do que com a quantidade de portas lógicas resultante, ou vice-versa.

Embora a fórmula de desempenho 3.1 estabelecida leve em consideração apenas dois parâmetros, ela pode ser estendida para incluir parâmetros adicionais, como consumo de energia por exemplo.

A quantidade de portas lógicas pode ser utilizada para estimar o número de recursos necessários numa possível implementação física do sistema modelado. No caso de uma implementação em um circuito integrado, por exemplo, a quantidade de portas lógicas pode ser utilizada para estimar o tamanho da área que será ocupada pelo sistema, e por conseqüência, determinar o custo de sua fabricação.

Por sua vez, a quantidade de níveis de lógica pode ser utilizada para determinar a velocidade de operação do circuito. No caso do roteador, pode ser utilizada inclusive para definir a taxa teórica máxima de vazão de dados. O roteador possui cinco portas de comunicação de entrada/saída, envia e recebe pacotes compostos por 32 bits, assim, uma estimativa da taxa teórica máxima de vazão do roteador pode ser equacionada da seguinte forma: $5 * 32 *$ velocidade de operação. Desta forma, as quantidades de portas lógicas e de níveis de lógica podem ser utilizadas para estimar importantes características do circuito implementado, como custo, velocidade de processamento e taxa de vazão.

\section{Resultados}

As fórmulas de desempenho e as fórmulas que indicam as quantidades de portas lógicas e de níveis de lógica dos decrementadores e do roteador foram descritas no MATLAB para a realização da análise comparativa da inserção desses decrementadores na arquitetura do roteador. A função desenvolvida gera dois gráficos referentes à comparação dos decrementadores. No primeiro gráfico, denominado Panorama 1, a função identifica o desempenho dos decrementadores sem levar em consideração a arquitetura do roteador na qual eles foram incluídos. A figura 2 mostra o gráfico do Panorama 1, considerando um peso de $70 \%$ em relação à quantidade de níveis de lógica, 30\% em relação à quantidade de portas lógicas e um tamanho de bloco contendo 4 bits. Em alguns decrementadores analisados, vetores de 4 bits foram agrupados em blocos de decremento. Desta forma, pode-se construir decrementadores híbridos, utilizando uma técnica de transporte internamente nos blocos de decremento e outra técnica de transporte entre os blocos. Sete tipo de decrementadores com técnicas diferentes para a obtenção dos bits de transporte foram analisados, quais sejam: transporte em cascata, transporte antecipado, uma modificação do transporte antecipado, transporte em cascata nos blocos de decremento e transporte selecionado entre os blocos (transporte cascata-selecionado), transporte antecipado nos blocos e transporte selecionado entre os blocos (transporte 
antecipado-selecionado), transporte antecipado modificado nos blocos e transporte selecionado entre os blocos (transporte antecipado modificado-selecionado) e, por fim, um decrementador com transporte antecipado nos blocos e transporte em cascata entre os blocos (transporte antecipado-cascata).

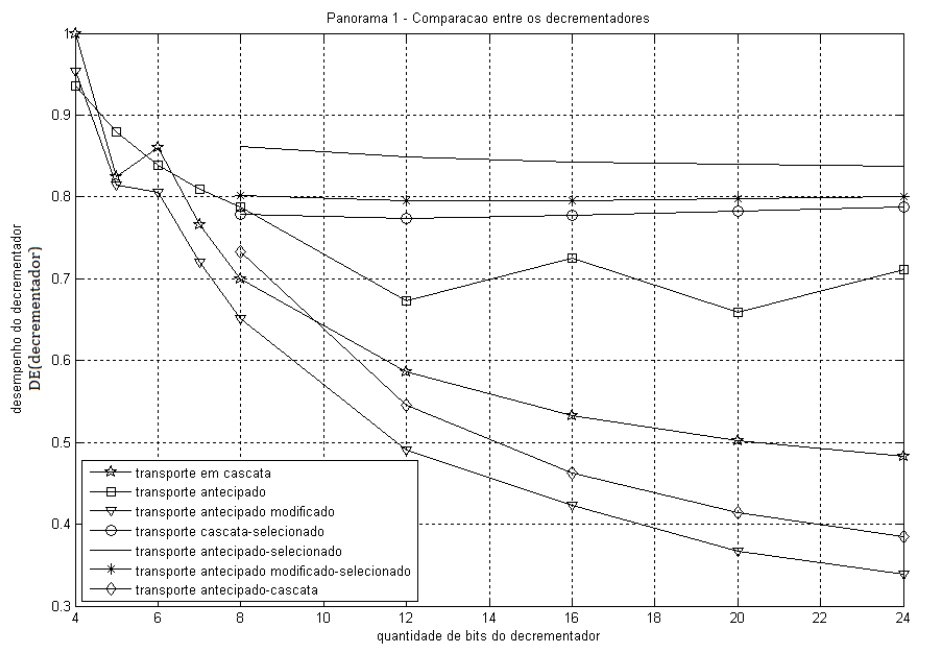

Figura 2: Panorama 1 - Comparação de desempenho entre os decrementadores

Pode-se notar que o decrementador com transporte antecipado-selecionado obteve o melhor desempenho entre 8 e 24 bits permanecendo na faixa de 0.8 e 0.9 de desempenho. Na faixa logo abaixo, entre 0.7 e 0.8 de desempenho, o segundo melhor desempenho foi do decrementador com transporte antecipado modificadoselecionado. O decrementador com transporte cascata-selecionado obteve a terceira melhor relação de desempenho. O pior desempenho foi do decrementador com transporte antecipado modificado atingindo um desempenho de aproximadamente 0.2 para 24 bits.

O segundo gráfico (Panorama 2), mostrado na figura 3, indica o desempenho da arquitetura do roteador projetada com cada um dos decrementadores.

Pode-se notar que o decrementador com transporte em cascata possui a melhor relação de desempenho entre 4 e 7 bits, obtendo o valor máximo, $D E=1$. A partir de 8 bits, três decrementadores permaneceram na faixa de desempenho entre 0.95 e 1.00. Todos os três possuem como base a técnica de transporte selecionado entre os blocos, se diferenciando com relação à implementação interna do bloco. Assim, o decrementador com transporte cascata-selecionado obteve o melhor desempenho. Seguido de perto, pelo decrementador com transporte antecipado modificado-selecionado. O terceiro melhor desempenho é do decrementador com transporte antecipado-selecionado. O pior desempenho entre 8 e 24 bits ficou com o decrementador com transporte em cascata, atingindo a marca de aproximadamente 0.55 para 24 bits. 


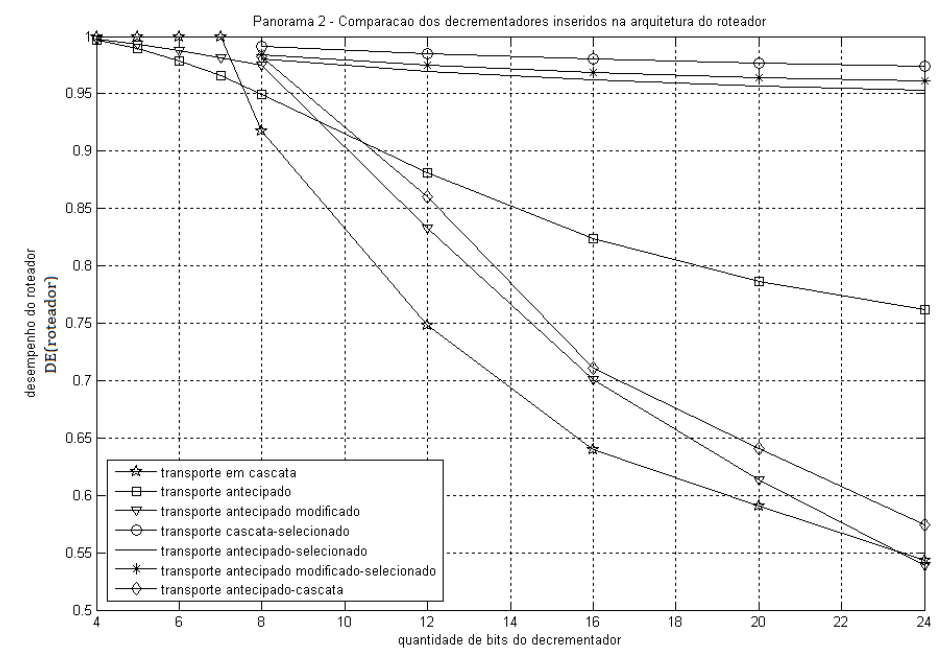

Figura 3: Panorama 2 - Desempenho do roteador com os decrementadores inseridos na arquitetura

\section{Discussão}

Como pode ser observado na figura 2, o decrementador com transporte antecipadoselecionado apresentou um desempenho melhor do que o decrementador com transporte cascata-selecionado. Porém, como mostra a figura 3, o roteador projetado com o decrementador com transporte cascata-selecionado obteve a melhor relação de desempenho, mesmo este decrementador tendo um desempenho individual inferior ao decrementador com transporte antecipado-selecionado.

Isso acontece porque na arquitetura do roteador, além de caminhos de dados existem também alguns caminhos relacionados ao controle da arquitetura. Com isso, a escolha de decrementadores mais rápidos pode melhorar o tempo de resposta de determinados caminhos de dados, mas contudo, pode não interferir no tempo de resposta dos caminhos de controle e de alguns caminhos de dados. Desta forma, apesar do decrementador com transporte antecipado-selecionado ser individualmente mais rápido, o desempenho em relação à quantidade de níveis de lógica do roteador projetado com qualquer um dos dois decrementadores será o mesmo pois o caminho crítico do roteador se mantém inalterado para os dois decrementadores. Contudo, o decrementador mais rápido que utiliza o transporte antecipado-selecionado gasta uma quantidade de portas lógicas um pouco maior do que o decrementador com transporte cascata-selecionado, e por este motivo a arquitetura do roteador utilizando o decrementador com transporte cascata-selecionado apresentou um desempenho melhor.

Também pode-se notar, na figura 3, que de modo geral os decrementadores que utilizam transporte selecionado entre os blocos apresentaram os melhores resultados. Convencionalmente, o somador com transporte selecionado tem um atraso relativamente pequeno, contudo, possui um número elevado de portas lógicas visto que, 
em cada bloco, dois somadores são necessários para produzir o resultado. Porém, o somador com transporte selecionado foi adaptado para o projeto do roteador (realizando apenas o decremento de um) e esta adaptação permitiu a inclusão de apenas um somador (decrementador) em cada bloco, reduzindo significativamente o número de portas lógicas. Com isso, estes decrementadores além de serem rápidos passaram a ter uma pequena quantidade de portas lógicas, tornando-se extremamente atrativos.

A escolha de um peso de $70 \%$ em relação à quantidade de níveis de lógica e de $30 \%$ em relação à quantidade de portas lógicas se deve aos motivos explicados na seqüência.

A velocidade de operação dos roteadores se torna muito importante visto que pacotes gerados pela arquitetura poderão atravessar vários roteadores até chegarem ao seus destinos finais. Se a velocidade de operação for muito baixa, os pacotes levarão muito tempo para ser entregues às transições do sistema e, por conseqüência, o tempo de resposta da arquitetura poderá aumentar muito. Assim, para se conseguir uma arquitetura eficiente, os roteadores que compõem o sistema de comunicação devem ser os mais rápidos possíveis. Porém, a quantidade de lógica utilizada para o projeto de cada roteador influencia no tamanho do chip a ser construído, o que acarretaria um custo maior na fabricação dessa arquitetura. Além disso, roteadores com uma quantidade muito grande de lógica tomaria muito espaço no chip, o que diminuiria a quantidade de circuitos que poderiam ser colocados na implementação física dessa arquitetura. Com menos circuitos no chip, o algoritmo de mapeamento seria capaz de alocar uma quantidade menor de lugares e transições das redes de Petri.

Contudo, foram realizadas análises utilizando outros valores para os pesos em relação às quantidades de portas lógicas e de níveis de lógica. Uma função em MATLAB foi especialmente desenvolvida para retornar o desempenho do roteador com cada um dos decrementadores variando os pesos em relação à porta lógica de $0 \%$ até $100 \%$, com uma taxa de incremento de $10 \%$. Para a maioria dos pesos em relação à quantidade de portas lógicas, de 0 até 0.8 , ou de $0 \%$ até $80 \%$, os três decrementadores que utilizam transporte selecionado entre os blocos apresentaram os melhores resultados. A partir de um peso em relação à quantidade de portas lógicas de aproximadamente $87 \%$, o decrementador com transporte em cascata ultrapassa o decrementador com transporte cascata-selecionado, se tornando o mais adequado. O decrementador com transporte em cascata possui uma quantidade reduzida de portas lógicas. Portanto, nesses casos, onde o nível de lógica não é quase levado em consideração, o decrementador com transporte em cascata leva maior vantagem apesar de apresentar o pior tempo de resposta.

\section{Conclusão}

Foram formuladas equações matemáticas que computam as quantidades de portas lógicas e de níveis de lógica de algumas estruturas aritméticas. Uma fórmula de desempenho foi desenvolvida para realizar uma análise comparativa da arquitetura do roteador com cada um dos decrementadores. O decrementador com transporte em cascata obteve a melhor relação de desempenho desde que seu tamanho esteja 
entre 4 e 7 bits. A partir de 8 bits, três decrementadores que utilizam a técnica de transporte selecionado obtiveram desempenho semelhante, se diferenciando com relação à implementação do bloco de subtração. Dos três, o decrementador com transporte cascata-selecionado obteve o melhor desempenho.

Todo projeto que possuir um sistema digital composto por decrementadores sem o bit de transporte final poderá fazer uso das fórmulas aqui desenvolvidas para definir a estrutura que melhor se adequará às condições estabelecidas pelo projetista. Desta forma, será possível automatizar o processo de identificação do circuito digital mais apropriado a ser incorporado em um determinado sistema. Assim, pode-se reduzir custo e economizar tempo na realização de um projeto, bem como auxiliar os projetistas na obtenção de sistemas com melhores desempenhos.

\begin{abstract}
A Network-On-Chip is under development as a reconfigurable hardware platform onto which one can directly implement Petri Nets. To determine the best subtractor architecture to be used with the network routers a series of equations were developed to compute the number of logic gates and of logic levels both of the subtractor and the router. A formula has been established to comparatively assess the performance of each subtractor when used with the router architecture.
\end{abstract}

Keywords. Router, subtractors, performance.

\title{
Referências
}

[1] K.M. Büyüksahin, F.N. Najm, High-level area estimation, em "ISLPED '02: Proceedings of the 2002 international symposium on Low power electronics and design", pp. 271-274, New York, NY, USA, 2002.

[2] J.P. Deschamps, G.J.A. Bioul, G.D. Sutter, "Synthesis of Arithmetic Circuits: FPGA, ASIC and Embedded Systems", Wiley-Interscience, New Jersey, 2006.

[3] D.S. Gelosh, D.E. Steliff, Modeling layout tools to derive forward estimates of area and delay at the rtl level, ACM Trans. Des. Autom. Electron. Syst., 5, No. 3 (2000), 451-491.

[4] G.D. Micheli, L. Benini, "Networks on Chips: Technology and Tools (Systems on Silicon)", Morgan Kaufmann Publishers, San Francisco, 2006.

[5] M. Nemani, F.N. Najm, High-level area and power estimation for vlsi circuits, em "ICCAD '97: Proceedings of the 1997 IEEE/ACM international conference on Computer-aided design", pp. 114-119, Washington, DC, USA, 1997.

[6] C. Ramachandran, F.J. Kurdahi, D.D. Gajski, A.C.-H. Wu, V. Chaiyakul, Accurate layout area and delay modeling for system level design, em "ICCAD '92: Proceedings of the 1992 IEEE/ACM International Conference on Computeraided design", pp. 355-361, Los Alamitos, CA, USA, 1992.

[7] A. Srinivasan, G. Huber, D. LaPotin, Accurate area and delay estimation from rtl descriptions, IEEE Transactions on VLSI Systems, 6, No. 1 (1998), 168-172. 